\title{
Local Statistics and Non-local Mean Filter for Speckle Noise Reduction in Medical Ultrasound Image
}

\author{
Jian Yang, Jingfan Fan, Danni Ai, Xuehu Wang, Songyuan Tang*, Yongtian Wang
}

Beijing Engineering Research Center of Mixed Reality and Advanced Display, School of Optics and Electronics, Beijing Institute of Technology, Beijing 10081, China

*Corresponding Author: sytang@ bit.edu.cn

\begin{abstract}
Medical ultrasound images are corrupted by speckle noise, which is multiplicative. This noise limits the contrast resolution in these images and complicates image-based quantitative measurement and diagnosis. In this study, the speckle noise in the ultrasound image is modeled by local statistics of the intensity distribution. And the non-local mean (NLM) filter is utilized to filter additional noise by applying the redundancy information in noisy images. A hybrid denoising method is proposed in consideration of the characteristics of both the local statistics of speckle noise and the NLM filter. The study combines local statistics with the NLM filter to reduce speckle in ultrasound images. The local statistics of speckle noise are estimated by local patches, while the intensity of the denoising pixel is computed by the weighted average of all the pixels by using the NLM. The weight is determined according to the similarity measures between the intensities of the local patches. The performance of the proposed method is evaluated on synthetic data, simulated images, and real images. Results of quantitative analysis and visual inspection of the synthetic data and of the real images demonstrate that the proposed method outperforms the original NLM, as well as many previously developed methods.
\end{abstract}

Key Words: Ultrasound image, Denoising, Speckle noise, Non-local means

\section{Introduction}

Ultrasound has been used to image the human body for over 60 years and is among the most widely used imaging technologies in medicine. Ultrasound is advantageous over existent non-invasive imaging techniques, such as CT, MRI, and PET, in that it is portable, inexpensive, free of radiation risk, and acquires images in real time. This imaging technology is widely used to visualize internal body structures, including tendons, muscles, joints, vessels, and internal organs for potential pathology or lesions. Ultrasound is also widely used to examine pregnant women.

An inherent characteristic of ultrasound imaging is the presence of speckle noise. Speckle is a random deterministic interference pattern that degrades the edges and fine details in an image. This phenomenon complicates the detection of small and low-contrast lesions. It also reduces the accuracy of ultrasound image-processing tasks such as feature extraction, segmentation, registration, and classification. To 
obtain reliable analysis and diagonal results, speckle reduction is a necessary preprocessing step in ultrasound image processing. Moreover, as the denoising technique can remove the speckle noise that may interfere the similarity measurement determination, it is also benefited for the $3 \mathrm{D}$ reconstruction procedures for accurately recovering the ultrasound volume data [1-3]. Therefore, the main objective of the current study is to remove speckle noise on ultrasound images.

Speckle noise can be removed by compounding [4], post-processing, or both [5]. The compounding method minimizes noises when images are acquired using many transducers. Similar regions of images acquired by each transducer are combined to form a final image with improved quality. The postprocessing method mainly includes the following two classes: (i) a spatial domain that is applied directly to the original image and (ii) a transform domain that is initially transformed into the frequency domain by fast Fourier transformation and is followed by denoising in the frequency domain. Many methods have recently been developed to eliminate speckle noise from medical ultrasound images in the spatial domain. These methods usually assume that this noise is modeled as multiplicative. Therefore, specific filters must be designed to reduce it. The most commonly used filters include the median [5, 6], Lee [7], Frost [8], and Kuan filters [9]. These filters are based on the multiplicative speckle model and on local statistics [10].

The median filter is a nonlinear filter that effectively removes impulsive noise. It also reduces speckle. This filter applies median intensity in a selected region as the output pixel value of the center position of the region. Lee filters are based on minimum mean-square error (MMSE) [11]. The smoothing degree of Lee filter is inversely proportional to the variance over a local region. If the variance is high, usually near the edge, then the smoothing process is not performed. Otherwise, smoothing is conducted. Frost filter is an adaptive and exponentially weighted averaging filter [12]. The weights are computed based on the ratio of the local standard deviation to the local mean of the degraded image. The pixel of interest is replaced by a weighted averaging value within the moving window. The weighting factors decrease with distance from the pixel of interest and increase when the variance within the window increases. Once the transformation of the multiplicative noise model into a signal-dependent additive noise model is combined with the minimum square error criterion, Kuan filter can generate the linear MMSE for an image that is corrupted with uncorrelated, image-dependent noise [13]. The form of Kuan filter is similar to that of Lee filter, but it uses a different weighting value. Based on the Lee and Frost filters, an adaptive speckle reduction filter is designed by classifying the pixels [14]. Although these methods reduce speckle 
noise effectively, they also erase weak and diffused edges.

To preserve edges in images, the anisotropic diffusion (AD) filter [15] is applied to suppress speckle noise. This filter is effective for images corrupted by additive noise. The nonlinear coherent diffusion filter [16] log-transforms multiplicative speckle noises in ultrasonic images into additive Gaussian noises. The speckle reducing AD (SRAD) filter [17] processes images directly to preserve useful information. This filter is based on a partial differential equation and on the MMSE, which can be related directly to Lee and Frost filters [17, 18]. The oriented SRAD filter [19] improves on the denoising results of the SRAD filter using the local directional variance of image intensity. These methods are iterative and can preserve the edges in images while reducing noise. However, many fine structures are removed during iterations. The squeeze box filter (SBF) was developed to enhance the contrast in B-mode ultrasound images with respect to decreasing pixel variations in homogeneous regions while maintaining or improving the differences in the mean values of distinct regions [20]. The smoothing parameter determines the amount of smoothing and is derived from a few functions of the local statistics. This filter not only generates high signal-to-noise ratio (SNR) value but also effectively preserves edges. Wavelet denoising methods are also used to reduce speckle. Moreover, fixed [21] and soft thresholds [22] are used to limit speckle noise. Artifacts are produced by these methods.

The aforementioned methods for speckle noise reduction are based on local information. A method based on non-local mean (NLM) filter has been proposed recently [23]. No assumptions are made about the location of the pixels used to denoise the pixel of interest. Each pixel of the image denoised with the NLM algorithm can represent the weighted average of all pixels in the noisy image when a Gaussian function is employed as the smoothing function. The NLM filter is an effective denoising method and has also been adapted to reduce speckle noise. Optimized Bayesian NLM (OBNLM) applies a Bayesian framework to derive an NLM filter, which is adopted for the speckle noise model [24]. A Pearson distance is introduced to compute the similarity between two patches. The method can improve speckle denoising. In the current paper, we introduce a hybrid method that combines the local property of the local statistics with that of the non-local property of the NLM method. The patches used in the NLM are pre-processed based on the local statistics. Then, the processed patches are used to compute the weights for the NLM. The performance of the proposed method is also compared with that of other methods (median, Lee, Frost, Kuan, SRAD, SBF, NLM, and OBNLM). Synthetic data, simulated images, and real images are used to evaluate this performance. 


\section{Methodology}

\subsection{Speckle noise model}

In many methods, the speckle noise in ultrasound images is approximately modeled to a multiplicative noise as follows [7-10, 14, 16, 17, 19, 21, 22]:

$$
g(\boldsymbol{x}) \approx f(\boldsymbol{x}) u(\boldsymbol{x})
$$

where $\boldsymbol{x}$ is the pixel position; $g(\boldsymbol{x})$ is the observed image; $f(\boldsymbol{x})$ is the original image; and $u(\boldsymbol{x})$ is a Gaussian noise.

This model is simple and is easily log-transformed into an additive noise. Hence, we obtain the following equation:

$$
\log (g(\boldsymbol{x})) \approx \log (f(\boldsymbol{x}))+\log (u(\boldsymbol{x}))
$$

Recently studies indicate that the general speckle noise model accurately represents the speckle noise in the ultrasound images. This model has been successfully applied in many studies [24, 25].

The general speckle noise model can be defined as follows:

$$
g(\boldsymbol{x})=f(\boldsymbol{x})+f(\boldsymbol{x})^{r} u(\boldsymbol{x})
$$

where factor $r$ is related to the hardware of the ultrasound devices and $u(\boldsymbol{x})$ is a zero mean Gaussian distribution. In B-mode ultrasound image study, $r$ is set to 0.5 because it represents ultrasound data well $[24,26]$. When $r$ is equal to 1 , the model is the multiplicative noise.

\subsection{Noise estimation using local statistics}

The noise-free image $f(\boldsymbol{x})$ can be estimated by the local linear MMSE as follows [9]:

$$
\hat{f}_{L L M M S E}(\boldsymbol{x})=E[f(\boldsymbol{x})]+\frac{\sigma_{f}^{2}(\boldsymbol{x})}{\sigma_{g}^{2}(\boldsymbol{x})}[g(\boldsymbol{x})-E(g(\boldsymbol{x}))]
$$

where $\hat{f}_{\text {LLMMSE }}(\boldsymbol{x})$ is the estimation of $f(\boldsymbol{x}) ; \sigma_{f}^{2}(\boldsymbol{x})$ and $\sigma_{g}^{2}(\boldsymbol{x})$ are the variances of $f(\boldsymbol{x})$ and $g(\boldsymbol{x})$, respectively; and $E[f(\boldsymbol{x})]$ and $E(g(\boldsymbol{x}))$ are the expectations of the $f(\boldsymbol{x})$ and $g(\boldsymbol{x})$, respectively.

Given that $u(\mathbf{x})$ is a zero-mean Gaussian noise as per the model in Eq. (3), we obtain

$$
E(g(\boldsymbol{x}))=E[f(\boldsymbol{x})]
$$

Hence, the variance $\sigma_{g}^{2}(\boldsymbol{x})$ can be defined as follows:

$$
\begin{aligned}
\sigma_{g}^{2}(\boldsymbol{x}) & =E\left(g^{2}(\boldsymbol{x})\right)-E(g(\boldsymbol{x}))^{2}=E\left(f(\boldsymbol{x})+f(\boldsymbol{x})^{r} u(\boldsymbol{x})\right)^{2}-E(f(\boldsymbol{x}))^{2} \\
& =\sigma_{f}^{2}(\boldsymbol{x})+\sigma_{u}^{2}(\boldsymbol{x}) E\left(f(\boldsymbol{x})^{2 r}\right)
\end{aligned}
$$

where $\sigma_{u}^{2}(\boldsymbol{x})$ is the variances of $u(\boldsymbol{x})$.

In addition, $\hat{f}_{L L M M S E}(\boldsymbol{x})$ is revised as follows: 


$$
\begin{aligned}
& \hat{f}_{L L M M S E}(\boldsymbol{x})=E(g(\boldsymbol{x}))+\frac{\sigma_{g}^{2}(\boldsymbol{x})-\sigma_{u}^{2}(\boldsymbol{x}) E(g(\boldsymbol{x}))}{\sigma_{g}^{2}(\boldsymbol{x})}[g(\boldsymbol{x})-E(g(\boldsymbol{x}))], \text { when } r=0.5 \\
& \hat{f}_{L L M M S E}(\boldsymbol{x})=E(g(\boldsymbol{x}))+\frac{\sigma_{g}^{2}(\boldsymbol{x})-\sigma_{u}^{2}(\boldsymbol{x}) E(g(\boldsymbol{x}))^{2}}{\left(1+\sigma_{u}^{2}(\boldsymbol{x})\right) \sigma_{g}^{2}(\boldsymbol{x})}[g(\boldsymbol{x})-E(g(\boldsymbol{x}))], \text { when } r=1
\end{aligned}
$$

where $E(g(\boldsymbol{x}))$ and $\sigma_{g}^{2}(\boldsymbol{x})$ are approximated by the local mean. Meanwhile, the variance can be defined as follows:

$$
\begin{gathered}
E(g(m, n)) \cong \frac{1}{\left(2 W_{x}+1\right)\left(2 W_{y}+1\right)} \sum_{i=-W_{x}}^{i=W_{x}} \sum_{j=-W_{y}}^{j=W_{y}} g(m+i, n+j) \\
\sigma_{g}^{2}(m, n) \cong \frac{1}{\left(2 W_{x}+1\right)\left(2 W_{y}+1\right)} \sum_{i=-W_{x}}^{i=W_{x}} \sum_{j=-W_{y}}^{j=W_{y}}[g(m+i, n+j)-E(g(m, n))]^{2}
\end{gathered}
$$

where $W_{x}$ and $W_{y}$ are the half-widths of the local windows.

$\sigma_{u}^{2}(\boldsymbol{x})$ can be estimated from the homogeneous regions in the image [27]. In the homogeneous regions, $\sigma_{f}^{2}(\boldsymbol{x})$ is equal to 0 and is represented as follows:

$$
\sigma_{f}^{2}(\boldsymbol{x})=E\left(f(\boldsymbol{x})^{2}\right)-[E(f(\boldsymbol{x}))]^{2}
$$

Therefore, in the homogeneous regions, we obtain the following equation:

$$
E\left(f(\boldsymbol{x})^{2}\right)=[E(f(\boldsymbol{x}))]^{2}=[E(g(\boldsymbol{x}))]^{2}
$$

where $\sigma_{u}^{2}(\mathbf{x})$ is represented as follows:

$$
\begin{gathered}
\sigma_{u}^{2}(\boldsymbol{x})=\sigma_{g}^{2}(\boldsymbol{x}) / E(g(\boldsymbol{x})), \quad \text { when } r=0.5 \\
\sigma_{u}^{2}(\boldsymbol{x})=\sigma_{g}^{2}(\boldsymbol{x}) / E\left(f(\boldsymbol{x})^{2}\right)=\sigma_{g}^{2}(\boldsymbol{x}) / E(g(\boldsymbol{x}))^{2}, \text { when } r=1
\end{gathered}
$$

The background areas of medical ultrasound images are used to estimate $\sigma_{u}^{2}(\boldsymbol{x})$.

\subsection{Non-local mean filter}

In the NLM filter, the estimated intensity $\operatorname{NLM}(g(i, j))$ of pixel $(i, j)$ is the weighted average of all of the voxel intensities in the noisy image, which is defined as follows:

$$
\operatorname{NLM}(g(i, j))=\sum_{\mathrm{l}=0}^{N_{x}-1} \sum_{m=0}^{N_{y}-1} w(i, j, l, m) g(l, m)
$$

where $N_{x}$ and $N_{y}$ represent the size of the image; $\operatorname{NLM}(g(i, j))$ is the intensity of the image at location (i, j) as estimated by the NLM filter; and $\mathrm{g}(l, m)$ is the intensity of the noisy image at location $(1, \mathrm{~m})$. Meanwhile, $\mathrm{w}(i, j, l, m)$ is a weighting coefficient and is defined as follows:

$$
\begin{gathered}
\mathrm{w}(i, j, l, m)=\frac{1}{\mathrm{z}(i, j)} \mathrm{e}^{-\frac{\mathrm{G}_{a}\left\|g\left(\mathrm{~N}_{i, j}\right)-g\left(\mathrm{~N}_{l, m}\right)\right\|^{2}}{\mathrm{~h}^{2}}} \\
0 \leq \mathrm{w}(i, j, l, m) \leq 1, \quad \sum_{\mathrm{l}=0}^{N_{x}-1} \sum_{m=0}^{N_{y}-1} w(i, j, l, m)=1
\end{gathered}
$$

where h is a degree of filtering (smooth parameter). $N_{i, j}$ and $N_{l, m}$ are the patches centered at voxels $(i, j)$ and $(l, m)$, respectively, and their sizes are denoted by $n_{x} \times n_{y} . \mathrm{G}_{a}$ is a Gaussian weighting 
function with zero mean and a is the standard deviation. a is usually set to 1 . Meanwhile, $\mathrm{Z}(i, j)$ is a normalizing constant and is defined as follows:

$$
\mathrm{Z}(i, j)=\sum_{\mathrm{l}=0}^{N_{x}-1} \sum_{m=0}^{N_{y}-1} \mathrm{e}^{-\frac{\mathrm{G} a\left\|g\left(\mathrm{~N}_{i, j}\right)-g\left(\mathrm{~N}_{l, m}\right)\right\|^{2}}{\mathrm{~h}^{2}}}
$$

To modify each noisy voxel $g(i, j)$, its neighborhood $g\left(\mathrm{~N}_{i, j}\right)$ is compared with other neighborhoods of noisy pixels $g\left(\mathrm{~N}_{l, m}\right)$ in the entire image. Much computation is required as a result. In consideration of computational efficiency, the search window is usually much smaller than the entire image and is represented by $s_{x} \times s_{y}$. The process also produces good results.

\subsection{Non-local mean filter combined with local statistics (NLMLS)}

To combine NLM with local statistics, the patches that are used to compute the weighting coefficient and the normalizing constant are not derived directly from the noisy images. The patches are first smoothed by local statistics. The weighting coefficient and the normalizing constant are modified as follows:

$$
\begin{aligned}
& \mathrm{w}^{\prime}(i, j, l, m)=\frac{1}{\mathrm{Z}(i, j)} \mathrm{e}^{-\frac{\mathrm{G}_{a}\left\|\hat{f}_{L L M M S E}\left(\mathrm{~N}_{i, j}\right)-\widehat{f}_{\text {LLMMSE }}\left(\mathrm{N}_{l, m}\right)\right\|^{2}}{\mathrm{~h}^{2}}} \\
& \mathrm{Z}^{\prime}(i, j)=\sum_{\mathrm{l}=0}^{s_{X}-1} \sum_{m=0}^{S_{y}-1} \mathrm{e}^{-\frac{\mathrm{G}_{a}\left\|\hat{f}_{L L M M S E}\left(\mathrm{~N}_{i, j}\right)-\widehat{f}_{L L M M S E}\left(\mathrm{~N}_{l, m}\right)\right\|^{2}}{\mathrm{~h}^{2}}}
\end{aligned}
$$

where $\mathrm{h}$ is the smooth parameter. And NLMLS is defined as follows:

$$
\operatorname{NLMLS}(g(i, j))=\sum_{\mathrm{l}=0}^{s_{x}-1} \sum_{m=0}^{s_{y}-1} \mathrm{w}^{\prime}(i, j, l, m) g(l, m)
$$

\section{Experimental results}

Four experiments are performed to test the proposed method. Experiment $\mathrm{I}$ is conducted for the synthetic data that produces noise through the general speckle model [Eq. (3)] under different noise levels. Experiments II and III are performed for simulated images that are produced through the method of Bmode ultrasound simulation [28] and through Field II [29, 30]. Experiment IV is conducted for real ultrasound images. The simulated image experiments use original images to produce the corresponding simulated images. Therefore, the results of the test methods are easily evaluated through mean-square error (MSE), SNR, and structural similarity index metrics (SSIM) [31]. We obtain:

$$
\begin{gathered}
\mathrm{MSE}=\frac{1}{N_{x} N_{y}} \sum_{i=0}^{N_{x}-1} \sum_{j=0}^{N_{y}-1}[f(i, j)-\hat{g}(i, j)]^{2} \\
\mathrm{SNR}=10 \log _{10} \frac{\frac{1}{N_{x} N_{y}} \sum_{i=0}^{N_{x}-1} \sum_{j=0}^{N_{y}-1} g^{2}(i, j)}{M S E}
\end{gathered}
$$




$$
\operatorname{SSIM}(\hat{g}, f)=\frac{\left(2 \mu_{\widehat{g}} \mu_{f}+c_{1}\right)\left(2 \sigma_{\widehat{g} f}+c_{2}\right)}{\left(\mu_{\widehat{g}}^{2}+\mu_{f}^{2}+c_{1}\right)\left(\sigma_{\widehat{g}}^{2}+\sigma_{f}^{2}+c_{2}\right)}
$$

where $f$ is the original image and $\hat{g}$ is the estimation of the original image from the noisy image. And the unit of SNR is decibel $(\mathrm{db}) . \mu_{\hat{g}}$ and $\mu_{f}$ are the averages of images $\hat{g}$ and $f$, respectively. $\sigma_{\hat{g}}^{2}$ and $\sigma_{f}^{2}$ are the variances of images $\hat{g}$ and $f$, respectively. $\sigma_{\hat{g} f}$ is the covariance of images $\hat{g}$ and $f . c_{1}=$ $\left(k_{1} L\right)^{2}$ and $c_{2}=\left(k_{2} L\right)^{2}$ are two variables that stabilize the division with a weak denominator. $L$ is the range of the pixel-values. The default $k_{1}$ and $k_{2}$ values are 0.01 and 0.03 .

MSE compares the similarity of the denoising image to the original image. It also indicates the difference between the compared images. A low MSE value indicates that the estimated image is closer to the original image and that the method performs well. SNR measures the relationship between the original image and the estimation error. This relationship is used to evaluate improvements in smoothing. A high value of SNR indicates the favorable performance of the test method. The SSIM index measures the similarity between two images. It is designed to improve on traditional methods, such as MSE, which are inconsistent with the perception of the human eye. Structural information is the idea that the pixels display strong inter-dependencies, especially when these pixels are spatially close. These dependencies carry important information regarding the structure of the objects in the visual scene. The maximum SSIM value is 1 , which is attainable only when two images are completely identical. Meanwhile, the NLMLS filter is compared with different filters when these data are utilized with these quality criteria.

The compared filters include median, Lee, Frost, Kuan, SRAD, NLM, and OBNLM.

In this study, the window size of the median, Lee, Frost, and Kuan filters measures $3 \times 3$. The iterations of the SRAD and SBF filters are 500 and 15, respectively. The patch size of the NLM, OBNLM, and NLMLS filters is $5 \times 5$, and the corresponding searching size is $11 \times 11$. The smooth parameters of NLM, OBNLM, and NLMLS filters are typically proportional to the noise level of the image. Different $\mathrm{h}$ values are tested in our experiments, and we select the $\mathrm{h}$ value that produces the largest SNR.

\subsection{Evaluation of the general speckle model}

In this section, the original synthetic image is shown in Fig. 1(a). This image includes a rectangle, an oval, a line, a triangle, and a cardioid. Given that various speckle removing methods use the multiplicative noise model [Eq. 1], the $\mathrm{r}$ of the general speckle model [Eq. 3] is set to 1 to produce noise that is similar to that produced by Eq. (1).

The different noise levels $(\sigma=0.2,0.4,0.6$, and 0.8$)$ are incorporated into the original image. An 
example of noise $(\sigma=0.6)$ is presented in Fig. 1(b). The corresponding results with $\sigma=0.6$ are depicted in Figs. 1(c)-1(k). Significant noise is observed in Figs. 1(c)-1(h), thus indicating that the results obtained with the median, Lee, Frost, Kuan, SRAD, and SBF filters are unsatisfactory. The results generated with NLM, OBNLM, and NLMLS in Figs. 1(i)-1(k) suggest that these filters perform well. However, Figs. 1(i) and 1(j) exhibit many artifacts. Fig. 1(k), which illustrates the result of the proposed NLMLS filter, displays the ideal image as per visual inspection.
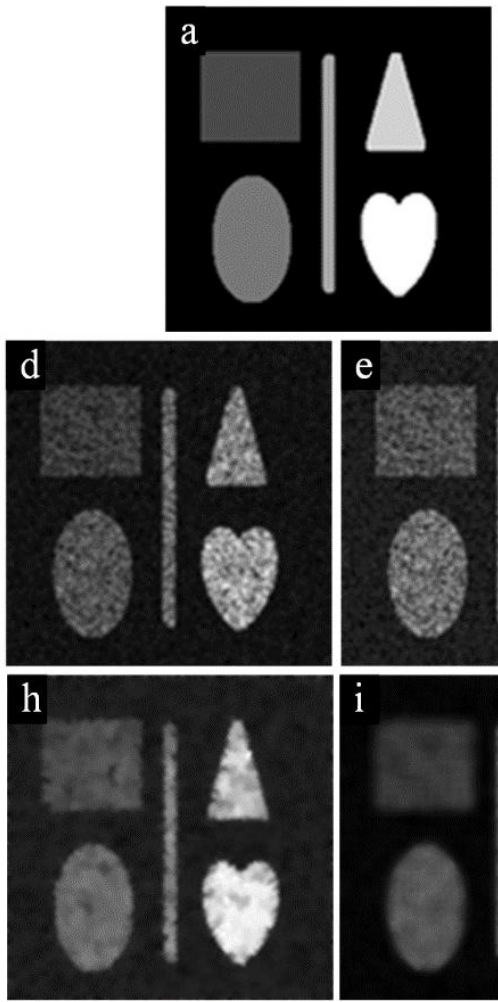
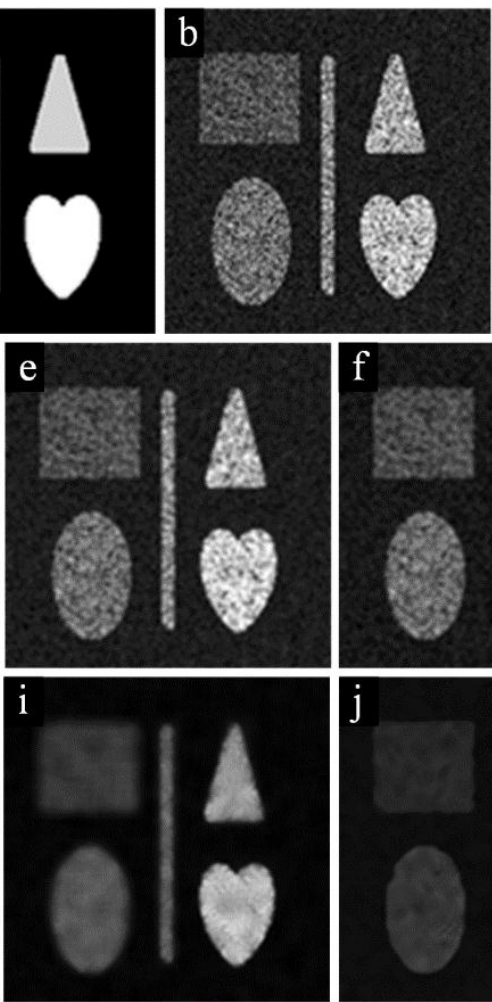
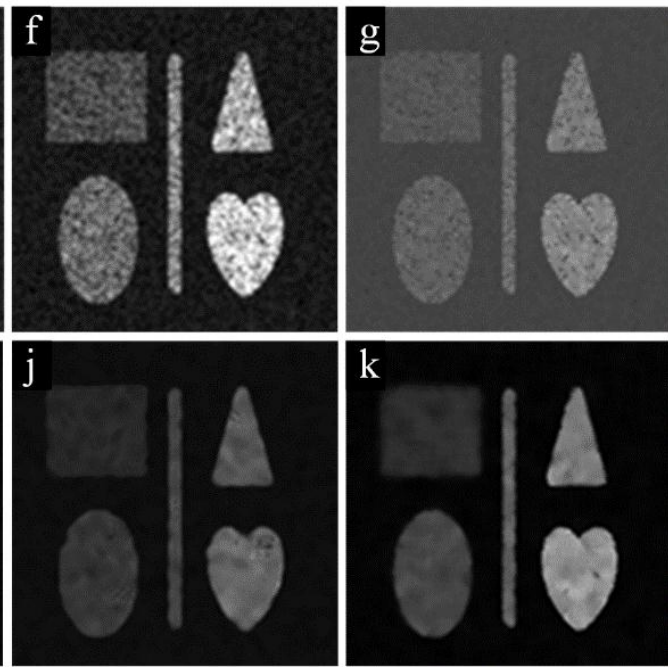

Fig. 1. Results of Experiment I: (a) original image, (b) image with speckle noise $\sigma=0.6$; results of (c) median filter, (d) Lee filter, (e) Frost filter, (f) Kuan filter, (g) SRAD filter, (h) SBF filter, (i) original NLM filter, (j) OBNLM filter, and (k) NLMLS filter.

The SNR, MSE, and SSIM values of test methods are calculated for comparison. The corresponding values are listed in Tables 1-3. Table 1 lists the SNRs under different noise levels. The maximum SNR value is represented in bold. NLMLS have maximum SNR at all noise levels. In terms of SNR, NLMLS is the best for various levels of noises. OBNLM is the second, and NLM is the third overall.

Table 2 lists the MSE with different noise levels, and the minimum MSE value is represented in bold. NLMLS has minimum MSE at all noise levels. In terms of MSE, NLMLS is the most effective, followed by OBNLM and NLM.

Table 3 lists the SSIM with different noise levels, and the maximum SSIM value is represented in 
bold. NLMLS has the maxmum SSIM at all noise levels. In terms of SSIM, the performance of NLMLS ranks first, that of OBNLM ranks second, and that of NLM ranks third overall.

Table 1. Comparison of SNR for the tested methods.

\begin{tabular}{c|c|c|c|c}
\hline SNR $(\mathrm{db})$ & $\sigma=0.2$ & $\sigma=0.4$ & $\sigma=0.6$ & $\sigma=0.8$ \\
\hline Noisy image & 17.18 & 11.26 & 8.25 & 6.23 \\
\hline Median & 23.55 & 18.25 & 15.78 & 14.18 \\
\hline Lee & 20.60 & 18.34 & 17.27 & 16.14 \\
\hline Frost & 19.67 & 15.95 & 13.18 & 11.05 \\
\hline Kuan & 20.49 & 18.21 & 17.05 & 15.84 \\
\hline SRAD & 28.10 & 21.99 & 13.19 & 8.96 \\
\hline SBF & 20.41 & 18.37 & 16.74 & 15.38 \\
\hline NLM & 26.92 & 20.06 & 17.08 & 15.85 \\
\hline OBNLM & 28.09 & 21.98 & 19.44 & 17.12 \\
\hline NLMLS & $\mathbf{2 8 . 9 1}$ & $\mathbf{2 3 . 2 0}$ & $\mathbf{2 0 . 3 1}$ & $\mathbf{1 8 . 3 3}$ \\
\hline
\end{tabular}

Table 2. Comparison of MSE for the tested methods.

\begin{tabular}{c|c|c|c|c}
\hline & $\sigma=0.2$ & $\sigma=0.4$ & $\sigma=0.6$ & $\sigma=0.8$ \\
\hline Noisy image & 226.3 & 939.0 & 2036.9 & 3621.2 \\
\hline Median & 50.1 & 168.1 & 295.1 & 427.7 \\
\hline Lee & 99.8 & 166.6 & 213.9 & 278.8 \\
\hline Frost & 124.3 & 297.6 & 575.5 & 970.9 \\
\hline Kuan & 102.3 & 171.7 & 225.4 & 299.3 \\
\hline SRAD & 17.8 & 73.3 & 580.9 & 1737.0 \\
\hline SBF & 102.7 & 169.0 & 248.7 & 326.8 \\
\hline NLM & 23.5 & 111.5 & 215.4 & 283.8 \\
\hline OBNLM & 17.9 & 72.7 & 130.5 & 223.8 \\
\hline NLMLS & $\mathbf{1 4 . 9}$ & $\mathbf{5 4 . 9}$ & $\mathbf{1 0 5 . 6}$ & $\mathbf{1 6 4 . 7}$ \\
\hline
\end{tabular}

Table 3. Comparison of SSIM for the tested methods.

\begin{tabular}{c|c|c|c|c}
\hline & $\sigma=0.2$ & $\sigma=0.4$ & $\sigma=0.6$ & $\sigma=0.8$ \\
\hline Noisy image & 0.58 & 0.32 & 0.21 & 0.16 \\
\hline Median & 0.91 & 0.78 & 0.67 & 0.58 \\
\hline Lee & 0.85 & 0.81 & 0.74 & 0.67 \\
\hline Frost & 0.75 & 0.70 & 0.41 & 0.31 \\
\hline Kuan & 0.83 & 0.77 & 0.70 & 0.63 \\
\hline
\end{tabular}




\begin{tabular}{c|c|c|c|c}
\hline SRAD & $\mathbf{0 . 9 8}$ & 0.89 & 0.47 & 0.27 \\
\hline SBF & 0.89 & 0.84 & 0.78 & 0.70 \\
\hline NLM & 0.94 & 0.88 & 0.83 & 0.79 \\
\hline OBNLM & 0.95 & 0.93 & 0.87 & 0.81 \\
\hline NLMLS & $\mathbf{0 . 9 8}$ & $\mathbf{0 . 9 4}$ & $\mathbf{0 . 8 9}$ & $\mathbf{0 . 8 8}$ \\
\hline
\end{tabular}

\subsection{B-mode ultrasound simulation}

A simulated image that possesses the property of B-scan images is produced. The method is based on theoretical tissue models and assumptions regarding the nature of pulse-echo imaging [28]. The statistical properties of a randomly inhomogeneous scattering medium are represented by the tissue model, whereas the ultrasonic pulse and beam shapes are simulated according to the nature of B-scan imaging. The simulated image is close to the clinical B-scan image as per visual inspection and exhibits many features that are similar to those of the clinical image.

The parameters of the B-mode scan are set as follows. The center frequency of the ultrasonic wave is $10 \mathrm{e} 6$. The velocity of sound in media is $1540 \mathrm{~m} / \mathrm{s}$. The variance of speckle distribution of the image is 0.01. The pulse-width of the transmitting ultrasonic wave is $2 \mathrm{~mm}$, and the beam-width of the transmitting ultrasonic wave is $1.5 \mathrm{~mm}$.

Fig. 2(a) shows the original image, which is the object in the simulation. The different regions of this image are regarded as different tissues. Following B-scan simulation, the simulated image is similar to the clinical image. Considering that the regions in the original image are large and uniform, those in the simulated image and the denoising image should be uniform as well. To evaluate the performance of the test methods apart from MSE, SNR, and SSIM, the sum of the variance (SV) of different regions is determined. SV can be defined as follows:

$$
\mathrm{SV}=\sqrt{\frac{\sum_{j=0}^{\mathrm{N}} \operatorname{var}(\text { region }(j))}{N}}
$$

where $\mathrm{N}$ is the number of the uniform region; $\mathrm{j}$ is the $\mathrm{j}$-th region; and $\operatorname{var}(\operatorname{region}(j))$ is the variance of the $\mathrm{j}$-th region. A low SV denotes a good result. The parameter $\mathrm{r}$ of the speckle noise model is set to 0.5 in this study because it represents ultrasound data well.

Figs. 2(c)-2(k) depict the results for (c) median, (d) Lee, (e) Frost, (f) Kuan, (g) SRAD, (h) SBF, (i) NLM, (j) OBNLM, and (k) NLMLS filters. The regions in Fig.s 2(c)- 2(h) are not uniform enough, but those in Figs. 2(i)-2(k) are more uniform and are very close. Nonetheless, Fig. 2(k) is slightly clear. A 
visual inspection confirms that the NLMLS filter performs best in this experiment.

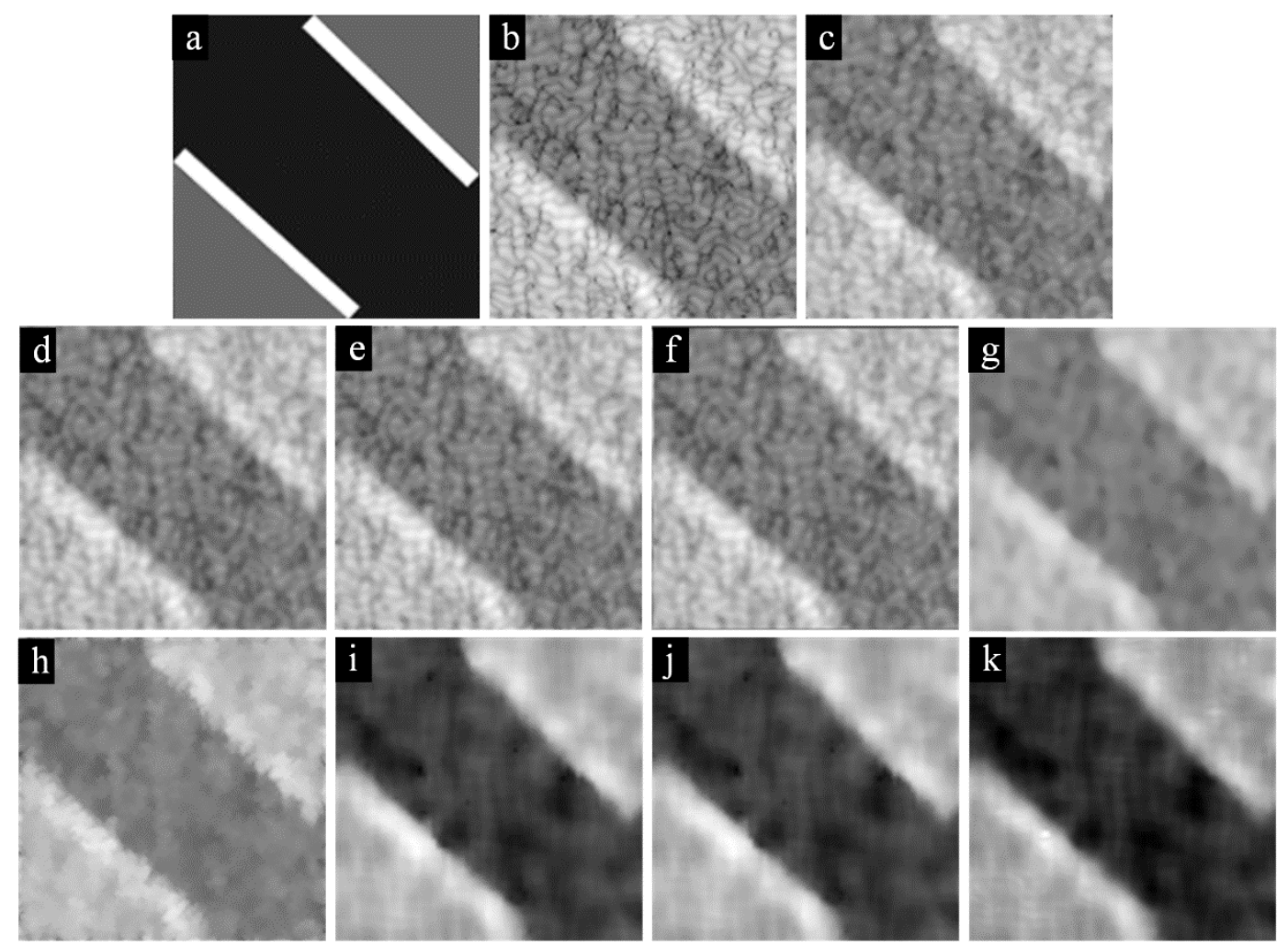

Fig. 2. Results of Experiment II: (a) original image, (b) image with speckle noise as simulated by theoretical tissue models based on assumptions regarding the nature of pulse-echo imaging; results of (c) median filter, (d) Lee filter, (e) Frost filter, (f) Kuan filter, (g) SRAD filter, (h) SBF filter, (i) original NLM filter, (j) OBNLM filter, and (k) NLMLS filter.

The values of SNR, MSE, SSIM, and SV are listed in Table 4. The maximum SNR and SSIM values are represented in bold, as are the minimum MSE and SV values. In terms of MSE, filter performance is ordered from best to worst as follows: NLMLS, Lee, Kuan, SRAD, OBNLM, Frost, NLM, median, and SBF. With respect to SNR, NLMLS is most effective among the filters. The performance of Lee, Kuan, and SRAD filters ranks second. That of Frost filter ranks fifth. NLM and OBNLM rank sixth. The median filter ranks eighth and SBF ninth. In terms of SSIM, NLMLS and OBNLM perform the best. NLM, SRAD, and SBF rank third, fourth, and fifth. Lee and the median filters ranked sixth. Kuan and Frost filters rank eighth and ninth. With regard to SV, filter performance is ordered from best to worst as follows: NLMLS, OBNLM, NLM, SRAD, SBF, Lee, Kuan, median, and Frost. Therefore, NLMLS is the best filter according to MSE, SNR, SSIM, and SV. It is followed by OBNLM and then NLM.

Table 4. Comparison of MSE, SNR, SSIM and SV of the testing methods.

\begin{tabular}{l|l|l|l|l}
\hline & MSE & SNR & SSIM & SV \\
\hline
\end{tabular}




\begin{tabular}{c|l|l|l|l}
\hline Noisy image & 9227.3 & 5.59 & 0.12 & 22.44 \\
\hline Median & 9161.7 & 5.63 & 0.28 & 14.94 \\
\hline Lee & 8964.1 & 5.67 & 0.28 & 14.67 \\
\hline Frost & 9069.0 & 5.65 & 0.18 & 18.40 \\
\hline Kuan & 8974.7 & 5.67 & 0.27 & 14.92 \\
\hline SRAD & 8976.6 & 5.67 & 0.34 & 13.15 \\
\hline SBF & 9415.1 & 5.54 & 0.31 & 13.87 \\
\hline NLM & 9070.1 & 5.64 & 0.38 & 11.97 \\
\hline OBNLM & 9025.3 & 5.64 & $\mathbf{0 . 3 9}$ & 11.61 \\
\hline NLMLS & $\mathbf{8 8 0 7 . 9}$ & $\mathbf{5 . 6 8}$ & $\mathbf{0 . 3 9}$ & $\mathbf{1 0 . 9 6}$ \\
\hline
\end{tabular}

\subsection{Field II simulation}

Field II is a program for simulating ultrasound transducer fields and ultrasound imaging using linear acoustics [29, 30]. The program calculates pulsed ultrasound fields with the Tupholme-Stepanishen method. An image acquired of a sample kidney in Field II is employed to produce the simulated ultrasound image. Fig. 3(a) depicts the original image, and Fig. 3(b) shows the simulating ultrasound image produced by Field II. Figs. 3(c)-3(k) illustrate the results for the median, Lee, Frost, Kuan, SRAD, SBF, original NLM, OBNLM, and NLMLS filters. The images in Figs. 3(c)-3(f) retain much noise. Those in Figs. 3(g), 3(h), and 3(j) are overly smooth. The images in Figs. 3(i) and 3(k) appear very close, but the latter is slightly sharper than the former. Therefore, the image in Fig. 3(k), which corresponds to that filtered with NLMLS in the experiment, is ideal as per visual inspection.
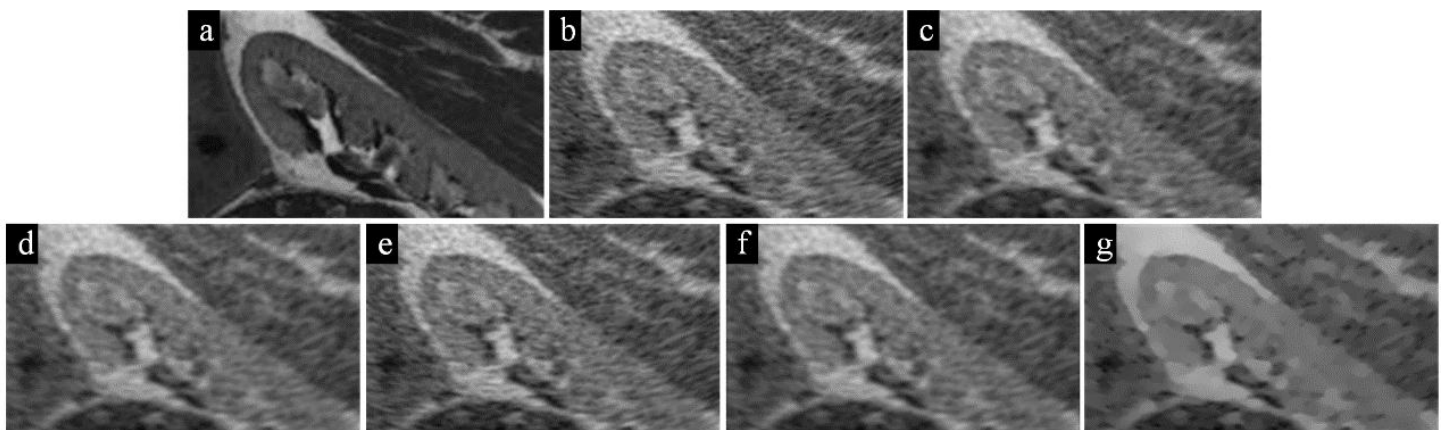

$\mathrm{h}$
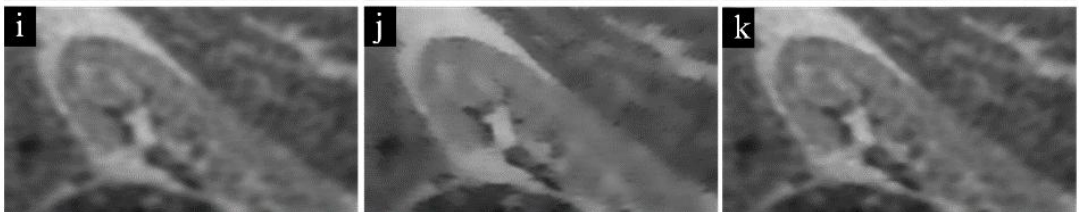

Fig. 3. Results of Experiment III: (a) original image, (b) image with speckle noise as simulated by Field II; results of (c) median filter, (d) Lee filter, (e) Frost filter, (f) Kuan filter, (g) SRAD filter, (h) SBF filter, (i) original NLM filter, (j) OBNLM filter, and (k) NLMLS filter.

Table 5 lists the MSE, SNR, and SSIM values of the test methods. The maximum SNR and SSIM 
values, as well as the minimum MSE value, are represented in bold. In terms of MSE results, NLMLS, OBNLM, and NLM rank first, second, and third. With regard to SNR results, NLMLS ranks first, followed by OBNLM and NLM at second. In terms of SSIM, NLMLS, OBNLM, and NLM are the most effective filters. Thus, NLMLS is the best filter based on MSE, SNR, and SSIM values. OBNLM and NLM are ranked second and third.

Table 5. Comparison of MSE, SNR, and SSIM for the testing methods.

\begin{tabular}{c|c|c|c}
\hline & MSE & SNR & SSIM \\
\hline Noisy image & 2443.7 & 8.54 & 0.13 \\
\hline Median & 2085.8 & 9.17 & 0.36 \\
\hline Lee & 1972.6 & 9.35 & 0.40 \\
\hline Frost & 2103.9 & 9.10 & 0.26 \\
\hline Kuan & 1945.5 & 9.37 & 0.39 \\
\hline SRAD & 1930.5 & 9.42 & 0.46 \\
\hline SBF & 2126.1 & 9.13 & 0.43 \\
\hline NLM & 1879.3 & 9.52 & $\mathbf{0 . 5 1}$ \\
\hline OBNLM & 1876.1 & 9.52 & $\mathbf{0 . 5 1}$ \\
\hline NLMLS & $\mathbf{1 8 2 2 . 2}$ & $\mathbf{9 . 6 1}$ & $\mathbf{0 . 5 1}$ \\
\hline
\end{tabular}

\subsection{Real image}

Given that the experiments on real images do not apply original images, the results of the tested methods are difficult to evaluate qualitatively. All of the clinical images used in this study are provided by the PLA General Hospital, Beijing, the size of the image is $256 x 256$. . The use of these data was approved by the institutional ethical review board of the university. The patients involved in our study have also provided written consent.

In the experiment, the model parameter $r$ is set to 0.5 . The results of Fig. 4(a) are also presented in Figs. 4(b)-4(h). These Figs. correspond to the results of the median, Lee, Frost, Kuan, SRAD, SBF, NLM, OBLM, and NLMLS filters. The images in Figs. 4(f), 4(g), and 4(i) are overly smooth. Those in Figs. 4(b)-4(e) retain much noise. The images in Figs. 4(h) and 4(j) are ideal, and Fig. 4(j) is slightly clear. 

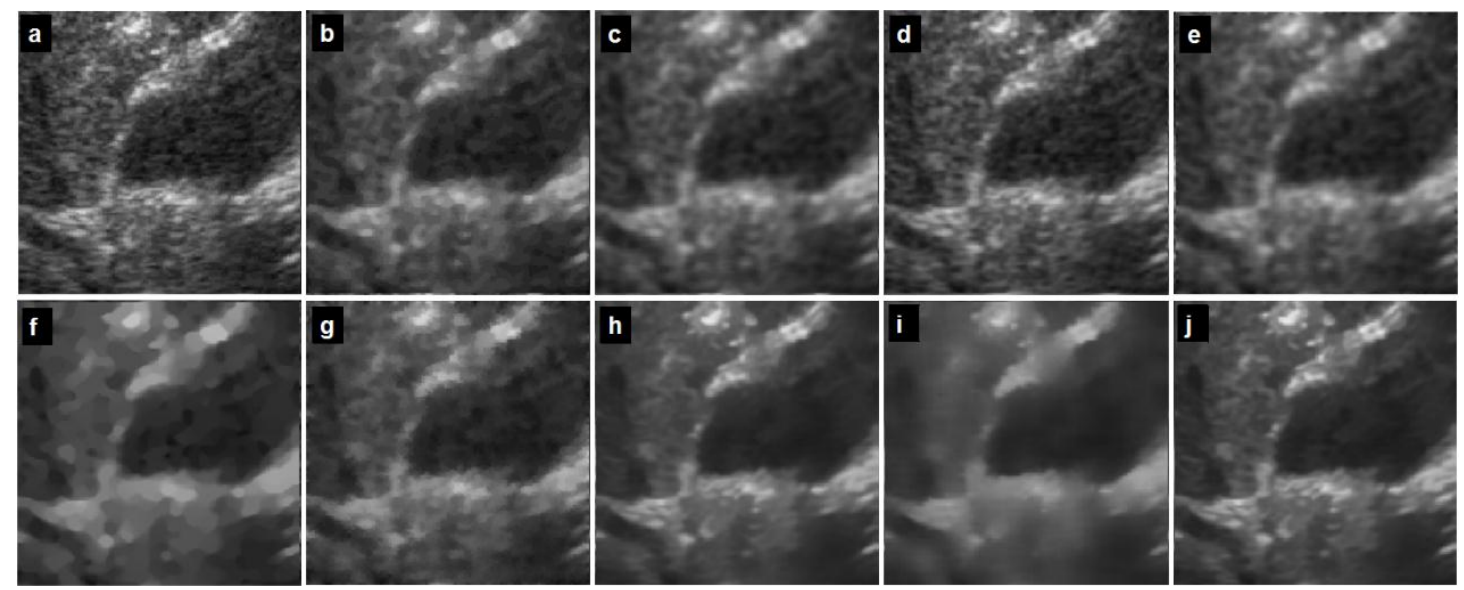

Fig. 4. Results of Experiment IV: (a) original image, results of (b) median filter, (c) Lee filter, (d) Frost

filter, (e) Kuan filter, (f) SRAD filter, (g) SBF filter, (h) original NLM filter, (i) OBNLM filter, and (j) NLMLS filter.

\section{Discussion and Conclusion}

In this study, an NLM-based filter was developed for ultrasound images by combining local information with non-local information to compute the similarity metrics of patches. Experiments were conducted on synthetic, simulated, and real images. The synthetic images were synthesized with different simulated speckle levels. The simulated images were subject to B-mode ultrasound simulation and Field II processing. Several denoising filters were compared through quantitative evaluations. The experimental results indicate that the proposed NLMLS exhibits the best quantitative measurements (SNR, MSE, SSIM, and SV). In the experiment of the simulated image constructed by the Field II, the SSIM values of NLM, OBNLM and NLMLS are equal (0.51) in table 5. The reason is that the simulated image, as illustrated in the Fig.3(b), has heavy noise that these three methods cannot differentiate the difference of SSIM. The visual inspection suggested that the performance of the proposed method is competitive with that of other methods. As per the experiments on real images, the results of NLMLS are the clearest after noise is smoothed.

\section{Acknowledgement}

This research was supported by the National Basic Research Program of China (2013CB328806), the Key Projects in the National Science \& Technology Pillar Program (2013BAI01B01), the National Hi- 
Tech Research and Development Program (2013AA013703). Thanks to Mr. Debdoot Sheet, who is a senior research fellow in Indian Institute of Technology Kharagpur, India, for providing us with the Matlab code for B-mode ultrasound image simulation.

\section{Reference}

[1] F. Lu, Y. Matsushita, I. Sato, T. Okabe, and Y. Sato, "Uncalibrated photometric stereo for unknown isotropic reflectances," in Computer Vision and Pattern Recognition (CVPR), 2013 IEEE Conference on, 2013, pp. 1490-1497.

[2] F. Lu, X. Ji, Q. Dai, and G. Er, "Multi-view stereo reconstruction with high dynamic range texture," in Computer Vision-ACCV 2010, ed: Springer, 2011, pp. 412-425.

[3] F. Lu, I. Sato, and Y. Sato, "Uncalibrated Photometric Stereo Based on Elevation Angle Recovery From BRDF Symmetry of Isotropic Materials," in Proceedings of the IEEE Conference on Computer Vision and Pattern Recognition, 2015, pp. 168-176.

[4] V. Behar, D. Adam, and Z. Friedman, "A new method of spatial compounding imaging," Ultrasonics, vol. 41, pp. 377-384, 2003.

[5] Y. Chen, S. L. Broschat, and P. J. Flynn, "Phase insensitive homomorphic image processing for speckle reduction," Ultrasonic imaging, vol. 18, pp. 122-139, 1996.

[6] J. L. Mateo and A. Fernández-Caballero, "Finding out general tendencies in speckle noise reduction in ultrasound images," Expert Systems with Applications, vol. 36, pp. 7786-7797, 2009.

[7] J.-S. Lee, "Digital image enhancement and noise filtering by use of local statistics," IEEE Transactions on Pattern Analysis and Machine Intelligence, pp. 165-168, 1980.

[8] V. S. Frost, J. A. Stiles, K. S. Shanmugan, and J. Holtzman, "A model for radar images and its application to adaptive digital filtering of multiplicative noise," IEEE Transactions on Pattern Analysis and Machine Intelligence, pp. 157-166, 1982.

[9] D. T. Kuan, A. A. Sawchuk, T. C. Strand, and P. Chavel, "Adaptive noise smoothing filter for images with signal-dependent noise," IEEE Transactions on Pattern Analysis and Machine Intelligence, pp. 165-177, 1985.

[10] S. Aja-Fernández, G. Vegas-Sánchez-Ferrero, M. Martín-Fernández, and C. Alberola-López, "Automatic noise estimation in images using local statistics. Additive and multiplicative cases," Image and Vision Computing, vol. 27, pp. 756-770, 2009.

[11] R. Sivakumar, M. K. Gayathri, and D. Nedumara, "Speckle filtering of Ultrasound B-scan images - A comparative study between spatial and diffusion filters," 2010 IEEE Conference on Open System, pp. 80-85, 2010.

[12] M. Raman and A. Himanshu, "Performance evaluation of various speckle noise reduction filters on medical images," International Journal of Recent Trends in Engineering, vol. 2, pp. 22-25, 2009.

[13] Z. Renqi, O. Wanli, and C. Wai-Kuen, "Image postprocessing by Non-local Kuan's filter " Journal of Visual Communication and Image Representation, vol. 22, pp. 251-262, 2011.

[14] A. Lopes, R. Touzi, and E. Nezry, "Adaptive speckle filters and scene heterogeneity," IEEE Transactions on Geoscience and Remote Sensing, vol. 28, pp. 992-1000, 1990.

[15] P. Perona and J. Malik, "Scale-space and edge detection using anisotropic diffusion," IEEE 
Transactions on Pattern Analysis and Machine Intelligence, vol. 12, pp. 629-639, 1990.

[16] K. Z. Abd-Elmoniem, A. Youssef, and Y. M. Kadah, "Real-time speckle reduction and coherence enhancement in ultrasound imaging via nonlinear anisotropic diffusion," IEEE Transactions on Biomedical Engineering, vol. 49, pp. 997-1014, 2002.

[17] Y. Yu and S. T. Acton, "Speckle reducing anisotropic diffusion," IEEE Transactions on Image Processing, vol. 11, pp. 1260-1270, 2002.

[18] J. Rogowska and M. E. Brezinski, "Evaluation of the adaptive speckle suppression filter for coronary optical coherence tomography imaging," IEEE Transactions on Medical Imageing, vol. 19, pp. 1261-1266, 2000.

[19] K. Krissian, C.-F. Westin, R. Kikinis, and K. G. Vosburgh, "Oriented speckle reducing anisotropic diffusion," IEEE Transactions on Image Processing, vol. 16, pp. 1412-1424, 2007.

[20] C. T. Peter, D. G. Christopher, and T. A. Scott, "Ultrasound despeckling for contrast enhancement," Transactions on Image Processing, vol. 19, pp. 1847-1860, 2010.

[21] S. Xiang and Y. Zhang, "Maximization of the signal-to-noise ratio for two dimensional medical ultrasound transducer sensitivity improvement by denoising wavelets," in Int. Conf. Biomed. Eng., Hong Kong, 1996.

[22] X. Zong, A. F. Laine, and E. A. Geiser, "Speckle reduction and contrast enhancement of echocardiograms via multiscale nonlinear processing," IEEE Transactions on Medical Imaging, vol. 17, pp. 532-540, 1998.

[23] A. Buades, B. Coll, and J.-M. Morel, "A non-local algorithm for image denoising," in Computer Vision and Pattern Recognition, 2005. CVPR 2005. IEEE Computer Society Conference on, 2005, pp. 60-65.

[24] P. Coupé, P. Hellier, C. Kervrann, and C. Barillot, "Nonlocal means-based speckle filtering for ultrasound images," IEEE Transactions on Image Processing, vol. 18, pp. 2221-2229, 2009.

[25] X. Hao, S. Gao, and X. Gao, "A novel multiscale nonlinear thresholding method for ultrasonic speckle suppressing," IEEE Transactions on Medical Imaging, vol. 18, pp. 787-794, 1999.

[26] T. Loupas, W. N. McDicken, and P. L. Allan, " An adaptive weighted median filter for speckle suppression in medical ultrasonic images," IEEE Transactions on Circuits and Systems, vol. 36, pp. 129-135, 1989.

[27] G. Torricelli, F. Argenti, and L. Alparone, "Modelling and assessment of signal-dependent noise for image denoising," 11th European Conference on Signal Processing, EUSIPCO, pp. 287-290, 2002.

[28] J. C. Bamber and R. J. Dickinson, "Ultrasonic B-scanning: a computer simulation," Physics in medicine and biology, vol. 25, 1980.

[29] J. A. Jensen and N. B. Svendsen, "Calculation of pressure fields from arbitrarily shaped, apodized, and excited ultrasound transducers," IEEE Transactions on Ultrasonics, Ferroelectrics and Frequency Control, vol. 39, pp. 262-267, 1992.

[30] J. A. Jensen, "Field: A program for simulating ultrasound systems," presented at the 10TH NORDICBALTIC CONFERENCE ON BIOMEDICAL IMAGING,, 1996.

[31] Z. Wang, A. C. Bovik, and H. R. Sheikh, " Image quality assessment: from error visibility to structural similarity," IEEE Transactions on Image Processing, vol. 13, pp. 600-612, 2004. 
Jian Yang received his Ph.D. degree in optical engineering from the Beijing Institute of Technology in 2007. He was a postdoctoral research fellow with the Mouse Imaging Centre, Hospital for Sick Children, Toronto, Canada, from 2007 to 2009. He is currently an associate professor with the School of Optoelectronics, Beijing Institute of Technology, China. He focuses his research interests on medical image processing, augmented reality

Jingfan Fan received his B.Sc. degree in optical engineering from the Beijing Institute of Technology in 2010. He is currently a Ph.D student, his research interests include medical image processing and augmented reality.

Ai Danni received the M.E. and B.E. degrees from Xi'an Jiaotong University in 2008 and 2005, and the D. E. degree from Ritsumeikan University, Japan, in 2011. Before joining Beijing Institute of Technology as a postdoctoral research fellow in December 2013, she was a researcher in Hitachi (China) Research \& Development Corporation, Shanghai since July 2012, and a postdoctoral research fellow in Ritsumeikan University and

Xuehu Wang received his B.Sc. degree in optical engineering from the Beijing Institute of Technology in 2010. He is currently a Ph.D student, his research interests include medical image processing and augmented reality.

Songyuan Tang received a B.S. degree in 1986 from Xiangtan University, China, a M.S. degree in 1991 from Sichuan University, China and a Ph.D. degree from the Institute of Automation, Chinese Academy of Science in 2004, China. He was a researcher at the college of Information Science and Engineering, Ritsumeikan University, Kyoto, Japan, in 2006, was a visiting scholar with the University of Pennsylvania from 2007 to 2008 and was a postdoctoral with the University of North Carolina at Chapel Hill from 2008 to 2009. He is currently an associate professor with the Institute of Beijing Technology, Beijing, China. His research interests include medical image analysis, intelligent signal and image processing,

Yongtian Wang received his B.Sc. degree in precision instrumentation from Tianjin University, China, in 1982, and his Ph.D. degree in optics from the University of Reading, England, in 1986. He is currently a Yangtze River Scholar of the Chinese Ministry of Education, a professor and the director of the Center for Research on Optoelectronics and Information Technology in Beijing Institute of Technology. His research interests include 


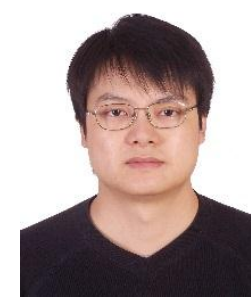

Jian Yang

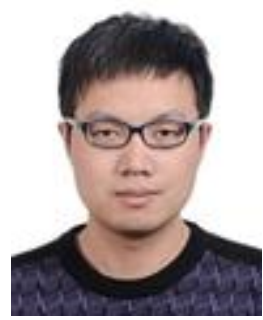

Jingfan Fan

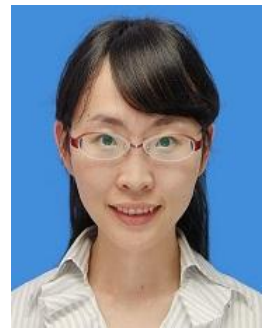

Ai Danni

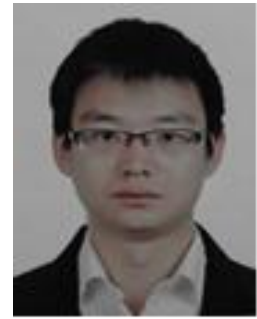

Xuehu Wang

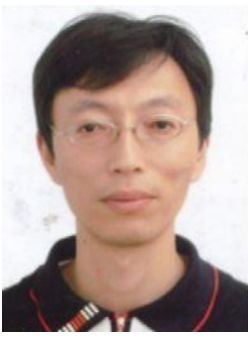

Songyuan Tang

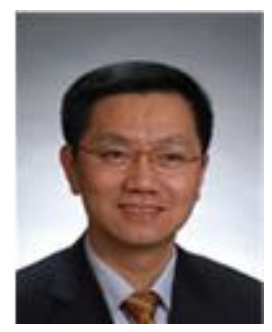

Yongtian Wang 